\title{
Modeling health oriented lifelong learning (HOLL) to prevent, delay and/or treat aging or aged dementias caused by less mentally stimulating activities
}

\author{
Cheng $\mathrm{K}^{*}$, Cheng $\mathrm{V}^{2}$ and $\mathrm{Zou}_{\mathrm{CH}} \mathrm{CH}^{1}$ \\ ${ }^{1}$ Biomedical and Biochemical Info physics, Science Research Institute, USA \\ ${ }^{2}$ Infectious Diseases, Brigham and Woman's Hospital, 75 Francis Street Boston, MA, USA
}

\begin{abstract}
It was estimated that 46.8 million people worldwide are living with dementia in 2015 . This number will almost double every 20 years, reaching 74.7 million in 2030 and 131.5 million in 2050 .

Dementia is not curable today. Even though some drugs have been used in clinic researches, the drugs have side effects.

Clinic studies show, people with fewer years of formal education are at higher risk for Alzheimer's and other dementias than those with higher years of formal education. These results make us believe education, learning, mentally stimulating activities and memories have correlations from each other.

In our previous studies, we defined memories as environmentally acquired information or signals (charges) stored or retained in the brain neurons (synapses). We modelled the long term memories (LTM) as consolidated (charged) proteins in the synapses. The charges may be discharged, therefore the memories may be lost because neurons are degenerated or died.

Here, we firstly clarify learning is to acquire new information, not to repeat old information. We propose models to describe mechanisms of dementias caused by less mentally stimulating activities, and models of the education and learning to prevent, delay and/or treat the dementias.

We believe the health oriented lifelong learning (HOLL) is the most effective way to precisely acquire the environmental information and maintain correlated memories in different knowledge fields as well as is a mental exercise that speeds up cerebral blood flow, nutrition supplies, O2 biochemical metabolism and produces the biological energy ATP and neuron chemic media in the synapses; and burn the over stored nutrients that may cause or induce the diseases.
\end{abstract}

The more complicate and/or versatile the learning is, the stronger and/or longer the acquired memories can be maintained.

Our HOLL models based on clinic trials data have not any side effect as long as the intensities are health oriented: moderate or mild.

\section{Introduction}

It was estimated that 46.8 million people worldwide are living with dementia in 2015. This number will almost double every 20 years, reaching 74.7 million in 2030 and 131.5 million in 2050 [1].

Dementia is not curable today. Even though some drugs have been used in clinic researches, the drugs have side effects [2].

Clinic studies show, people with fewer years of formal education are at higher risk for Alzheimer's and other dementias than those with higher years of formal education [2-7]. Some scientists believe, the less the mentally stimulating activities, the more likelihood the dementias [8-12]. These make us believe education, learning, mentally stimulating activities and memories have correlations from each other.

To cure or treat the aging or aged dementias, clinical researches have been performed using music or song (sing) therapies with no side effects [13], and using electric stimulation with minimum side effects [14,15].

On the other hand, clinical trials showed, bilingualism delays age at onset of dementias, independent of education and immigration status [16], or lifelong bilingualism maintains neural efficiency for cognitive control in aging bilingualism [17].
However, we have not found any published models or theories to depict the mechanisms of the clinical researches, and/or to prevent or delay the diseases, in a perspective of biomedical and biochemical info physics [18].

We previously proposed models to investigate Alzheimer's disease caused by neuron degeneration [19] and neuronal signal processes [20] based on theories of electromagnetic fields, in a perspective of biomedical and biochemical info physics [18].

Here, we firstly clarify learning is to acquire new information, not to repeat old information. We propose models to describe mechanisms of dementias caused by less mentally stimulating activities, and models of the education and learning to prevent, delay and/or treat the dementias in a perspective of biomedical and biochemical info physics.

${ }^{*}$ Correspondence to: Kang Cheng, Biomedical and Biochemical Info physics, Science Research Institute, 205 Hana Road, Edison NJ 08817, USA, Tel: 732-2480790, E-mail: kangcheng.1@netzero.net

Key words: clinical, biochemistry, O2, blood, correlated memories

Received: July 17, 2018; Accepted: July 19, 2018; Published: August 06, 2018 

activities

\section{Methods}

We use published clinic trial data [1-17] and our previous published models of brain memories [19,20], meridian channels [21,22] as well as theories of biochemistry, learning $[23,24]$ and artificial neural network $(\mathrm{ANN})[25,26]$.

\section{Modelling Results}

\section{Learning to build up and maintain brain memories}

We believe, for brain recognition, cognition, classification and signal responses: information retrieving or recalling is mostly from long, intermediate or short terms of memories (LTM, ITM or STM) via brain circuits or networks; information processing occurs mostly in the working memories (WM) in working cortices.

For dementia, LTM, ITM or STM are abnormal or nothing, working memories have poor or no cognitions, and/or the circuits are dysfunction [19].

We think, for most aging or aged patients with dementias, their brain neural networks or memories are degenerated, damaged or dysfunctional $[19,20]$. Therefore, we focus on modeling the prevention or postponement of degeneration of memories and circuits caused by less mentally stimulating activities in this paper.

In our previous studies $[19,20]$, we defined memories as environmentally acquired information or signals (charges) stored or retained in the brain neurons (synapses). We modelled the long term memories (LTM) as consolidated (charged) proteins in the synapses. The charges may be discharged, therefore the memories may be lost because neurons are degenerated or died.

In this study, we firstly clarify learning is to acquire new (not known, not familiar before) information, not to repeat old information. we believe the health oriented lifelong learning (HOLL) is the most effective way to precisely acquire the environmental information and maintain correlated memories in different knowledge fields as well as is a mental exercise that speeds up cerebral blood flow, nutrition supplies, $\mathrm{O} 2$ biochemical metabolism and produces the biological energy ATP and neuron chemic media in the synapses; and burn the over stored nutrients that may cause or induce the diseases.

Normally, a human brain has only $2 \%$ of the total body weight; however it takes $15 \%$ of the total blood flow and costs $20 \%$ of the total O2 supply [27].

Similar to do physical excises that intensifies motor and muscle functions of forces and dexterities, we believe, learning (a mental exercise) enhances neuron functions of brain memories and circuits; the more complicate and/or versatile the learning is, the stronger and/ or longer the acquired memories can be maintained.

Importantly, health oriented lifelong learning (HOLL) has not any side effect in clinical trials as long as the intensities are health oriented: moderate or mild.

The principle of our models is, how we can acquire the memories, we can keep or retain them: use health oriented lifelong learning (HOLL) to build up and maintain multiple correlated memories in different knowledge fields.

\section{Recalling or retrieving memories}

(Figure 1) shows our simplified model to depict a mechanism to recall or retrieve specific information from long term memories
(LTM) to working memories in working cortices based on published neuroscience data or models [28]. We assume the long term memories have been built up in related neurons (synapses) with conditional or unconditional stimuli and responses before.

(Figure 2) demonstrates our simplified model of retrieving two correlated memories of a concept of octave in two different knowledge fields. The concept can be in chemical theories [29] periodic table of elements and/or in music theories: periodic pitches. Obviously, if there

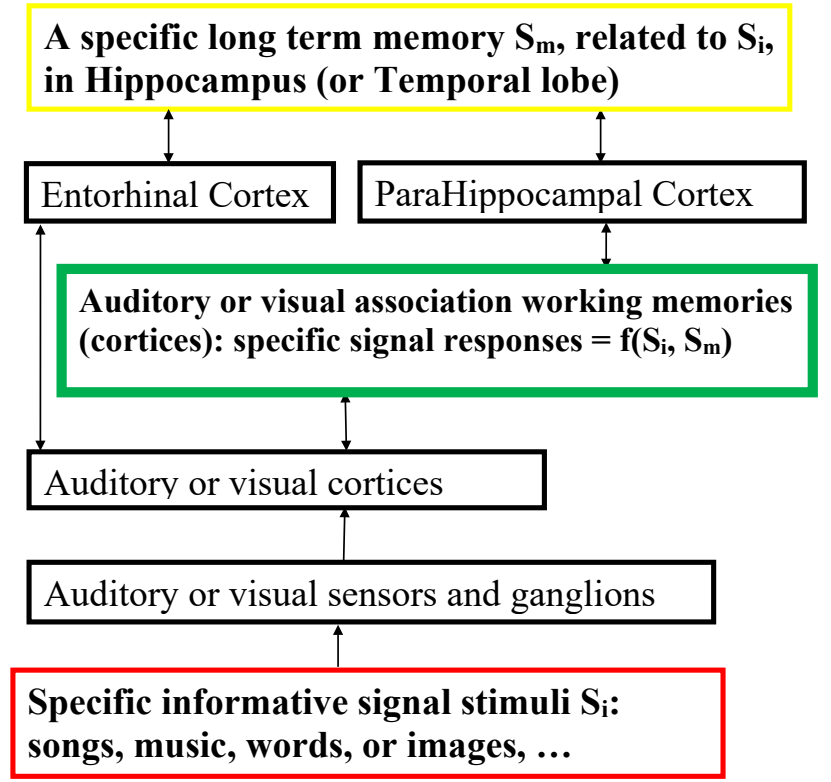

Figure 1. A simplified model of auditory or visual stimulation to retrieve or recall specific long term memories. $\mathrm{S}$ denotes a signal vector, subscript $\mathrm{i}$ and $\mathrm{m}$ respectively denote inputs and memories, arrow lines denote interactive circuits [26]

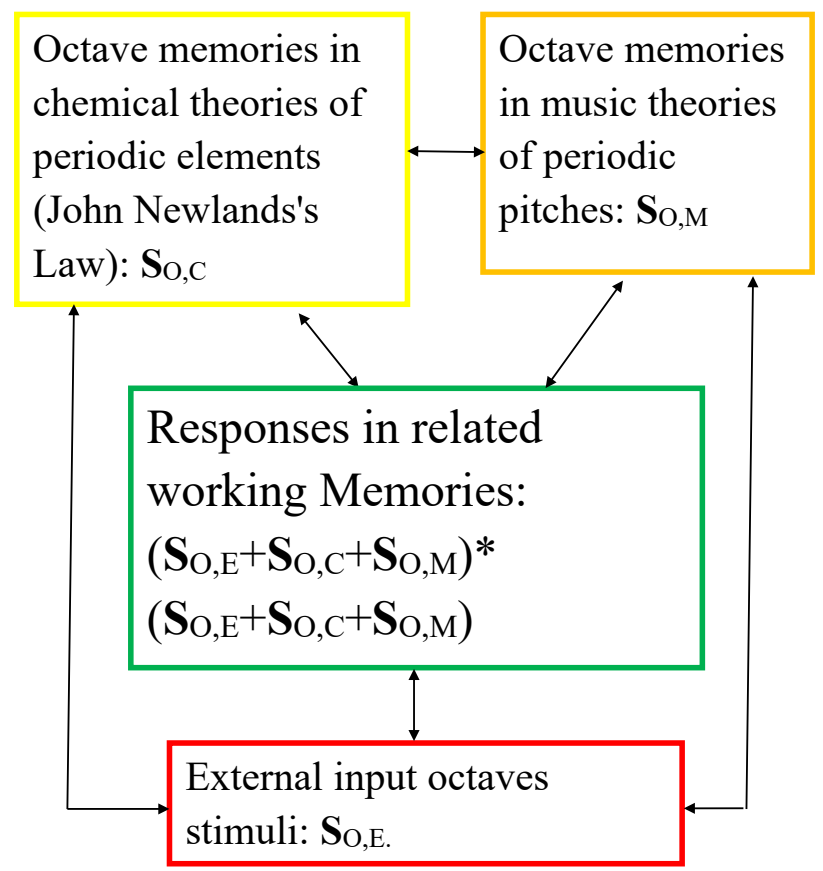

Figure 2. A simplified model of retrieving or recalling two correlated octave memories in two different knowledge fields: signal stimuli and responses, where * denotes a correlation operation. A more detail stimulation model is shown in Figure 1. Others are the same as that in Figure 1 

activities

is not any octave memory (stored signal or information in the brain), there will be only the autocorrelation of external input signals, and the neurons will have no idea what an octave is, though they have the correspondent recalling ability; if there is only one octave memory and a correspondent retrieving ability, there will be a correlation between the input and stored signals as well as the autocorrelation of the input signals; if there are two octave memories and correspondent retrieving abilities, there will be correlations between the input and stored signals and between the stored signals as well as autocorrelation of the input signals. The correlation between the memories represents an association [18-20]. We think the association can produce creative thinking.

Therefore, when performing signal stimulation, we believe the more memories with the same, similar or related concepts, the stronger the signal responses and the richer the association in the related working memories [18-20] and the less the probability that aging or aged dementias occur.

Figure 3 illustrates our simplified model of retrieving multiple correlated memories in different knowledge fields. The working memories can access the all of the long term memories [18-20]. Additionally, if one memory is incomplete, fuzzy or has errors, other correlated memories can accomplish, clear or correct cognitions or recognitions. The brain correlated memories can also explain why and/ or how normal brains do not loose memories after some neurons die because of metabolisms or other reasons.

Generally (Figures 2 \& 3), we think, cross correlated responses and related circuits are obtained by interactions between different signal stimuli or memories; while auto correlated responses and related circuits are obtained by interactions between the same or similar stimuli or memories [20]. The cross correlations involve conceptual and associative thinking; the auto correlations mostly involve materials and feelings. We believe the cross correlations can construct more complicated memories and circuits than the auto correlations do in our brain.

From the above models, we can extend, if a concept is stored in different knowledge fields of multiple languages, such as bilinguals, the responses or recognition signals will be stronger than that of monolinguals. Therefore, our models can explain why or how bilingualism can prevent, delay and/or treat (onsets of) dementias $[16,17]$, in a perspective of biomedical and biochemical info physics.

\section{To prevent or delay aging or aged dementias caused by less} mentally stimulating activities

We believe, except initial learning, learning is usually involved in recalling or retrieving old memories and circuits as well as building up new memories and circuits, because the acquiring knowledge frame will be constructed based on the acquired knowledge frame. The acquiring knowledge can be wider or deeper than the acquired knowledge. Therefore, learning refreshes or updates the old memories and circuits as well as constructs the new memories and circuits.

It is well known that mathematics and computer sciences, physics, chemistries and engineering are math oriented knowledge; literature, history, languages, and arts are word oriented knowledge. Math oriented learning can be used to mostly train space time imagination, logical reasoning, deducting and inducing.

We think, the math oriented learning can enhance or build up complex brain circuits and memories (neural networks), so that the networks can learn association and can help to produce creative thoughts as well as can construct more complex brain neural networks.
Figure 4 depicts our model of the brain (mental) activities of knowledge. Generally or usually, it is not very hard for mathematic, scientific or engineering people to learn liberal arts, oppositely, it is hard or very hard for liberal or artistic people to learn mathematics, sciences or engineering, because books (or knowledge) of mathematics and computer science, physics, chemistries, and engineering include words,

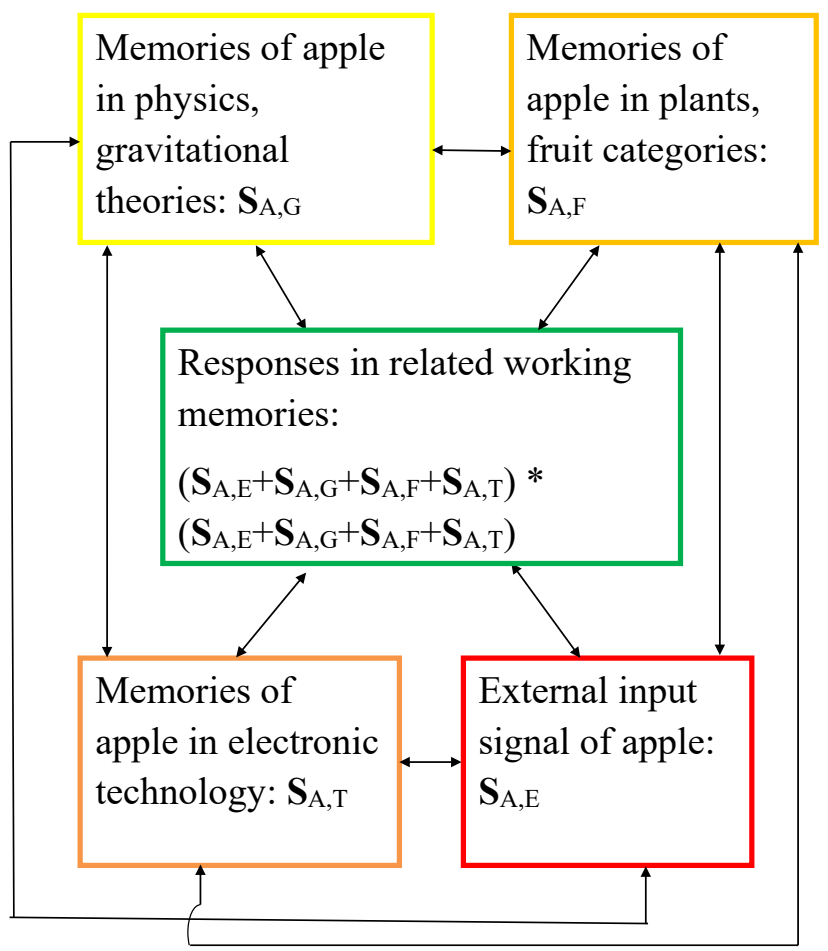

Figure 3. A simplified model of retrieving or recalling multiple correlated apple memories in different knowledge fields: signal stimuli and responses. Others are the same as that in Figure 1 or Figure 2

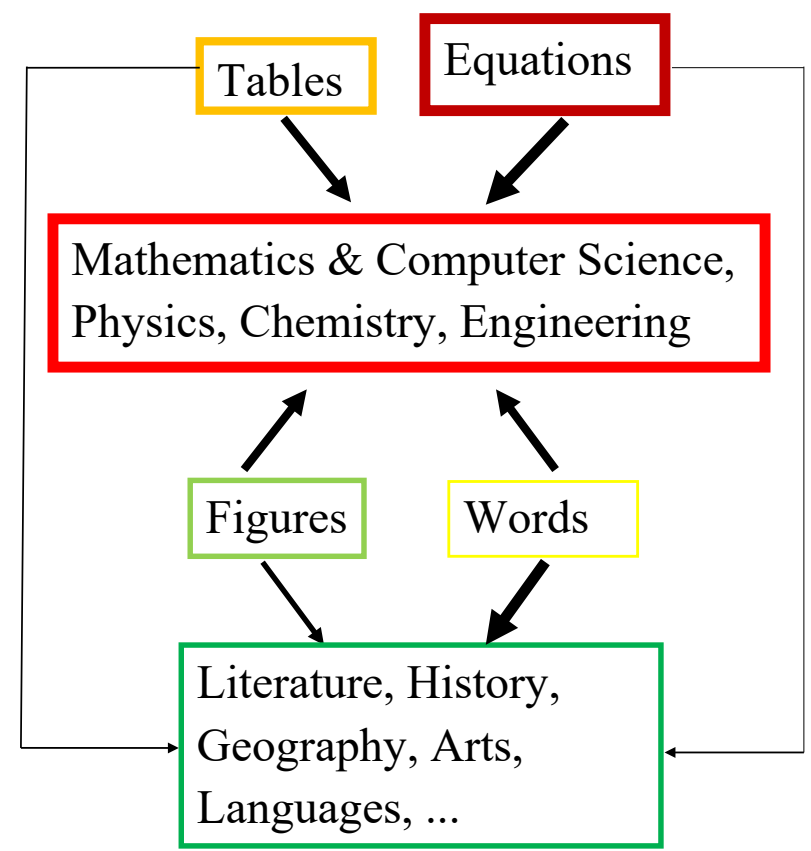

Figure 4. A model of the brain mental activities: The thicker the frame lines, the complex the activities; the thicker the arrows, the heavier the using weights 

activities

figures and complicated tables and equations; books (or knowledge) of biology and medicine include words, figures and simple tables and equations; books (or knowledge) of literature and arts include words and figures only.

Therefore, people also learn, update, refresh or review word oriented knowledge when they learn math oriented knowledge; oppositely, people (almost) do not learn, update, refresh or review math oriented knowledge when they learn word oriented knowledge.

We believe frequent learning (including practicing) of new knowledge of mathematics, physics, chemistries and engineering can help learners to develop or maintain complicated brain function. The frequent learning of the new knowledge could make optimum results to prevent, delay and/or treat (onsets of) dementias. Therefore, we suggest people frequently learn appropriate levels of mathematics, physics, chemistries, engineering or foreign languages as well as perform some abstract or logic thinking as much as they can and as often as possible.

We also believe the best way to learn knowledge (Figure 5) is to hear, write/draw, read/speak/sing and watch simultaneously.

Based on our model in (Figures 3-5), for the health oriented learning, we suggest aging or aged people to progressively study moderate challengeable, interesting, and versatile knowledge, such as mathematics (including computer sciences), sciences, engineering, foreign languages, arts and literatures.

A fast way to obtain the memories is to learn new knowledge progressively at schools, where complete and organized training sequences are available. Therefore, we think, to prevent or to delay aging or aged dementias caused by less mentally stimulating activities, back school programs are the best education procedures to most aging or aged people, especially, to pure physical or labouring workers who do not involve much brain (mental) activities. In the education programs, they start a teacher supervised learning [22], not only they can learn new knowledge, proceeding in an orderly way and step by step, with optimum learning laws, speeds, stabilities, challenge abilities, interests or curiosities, and performances, but also they can refresh the old memories, such as memories of old teachers, school mates and other related people and things, to keep their memories sharp.

After the supervised learning procedure, the aging or aged people can be transferred to a partial supervised and partial unsupervised learning procedure.

Finally, an (almost) unsupervised learning can be their lifelong learning procedure [16].

We can design all of the learning procedures are health oriented and very helpful to prevent, delay and/or treat (onsets of) aging dementias caused by less mentally stimulating activities; they can be also used to cure or treat the diseases.

To treat aging or aged dementias caused by less mentally stimulating activities

To cure patients with dementias, who have lost most of their memories, we think we should teach them like to educate children or babies, to build up their memories from the beginning.

\section{Mechanisms of clinical researches}

We can use the model in (Figure 1) to treat aging or aged patients with dementias caused by less mentally stimulating activities using repeated specific auditory (music) or visual signals (images) to recall specific memories by enhancing or regenerating specific circuits and/ or memories [24]. The responses in the related working memories are also specific. This model is our first priority to recommend to clinical trials. The repeated signals work like mental excises to intensify the brain circuits or memories.

Figure 6 demonstrates a simplified model of specific auditory (music) or visual (images) signals plus distributed electric stimulation through meridian channels $[21,22]$ to retrieve long term memories. The electric stimulation works like distributed mental excises to enhance

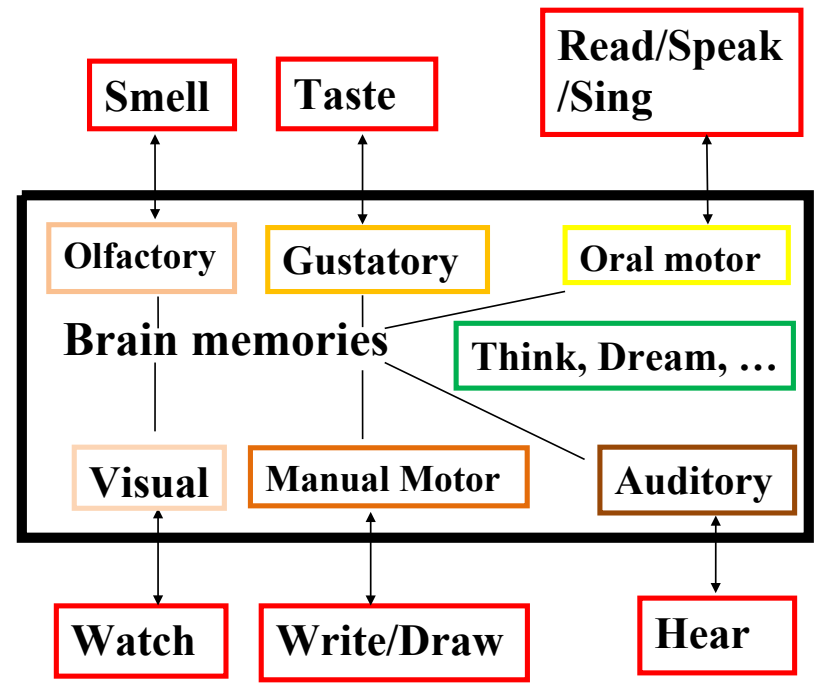

Figure 5. A simplified model to learn new concepts and to buildup correlated memories, e.g., apples, by smelling, tasting, reading/speaking/sing, watching, writing/drawing, and hearing. The linkages of brain neural networks are omitted

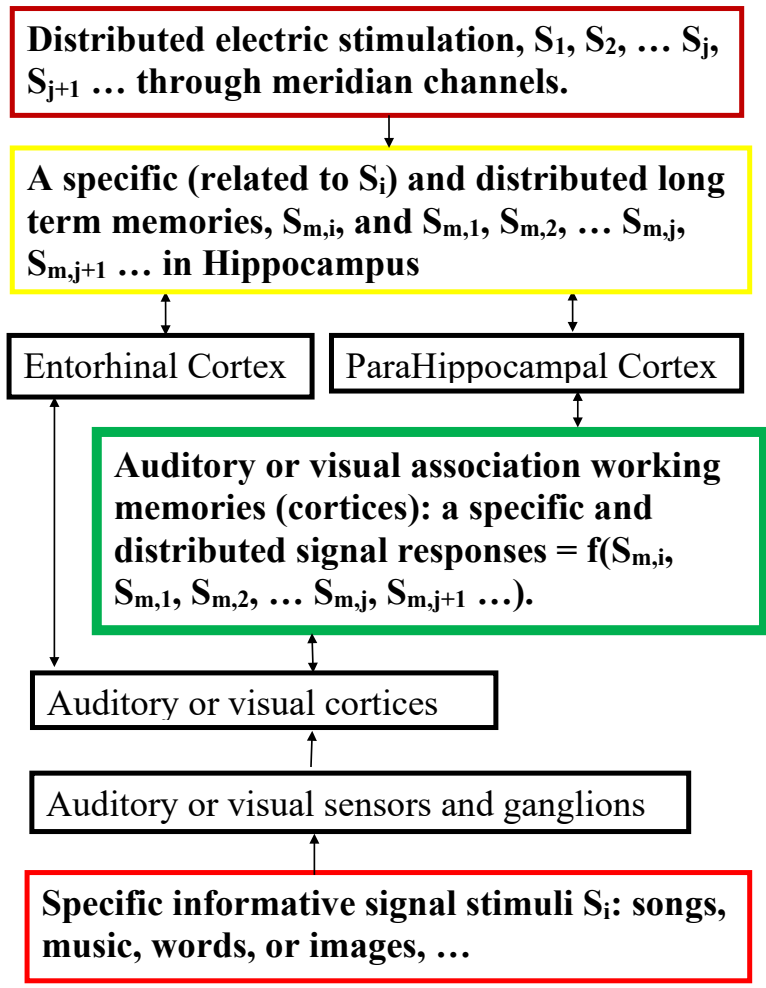

Figure 6. A simplified model of auditory or visual plus distributed stimulation to recall or retrieve long term memories 

activities

distributed circuits or/and memories. However, the responses in the related working memories are distributed too. Therefore, our model in this figure gives an explain the clinical researches [14,15]: the distributed stimulation improves the patient's recalling abilities, but may disturb normal people's retrieving memories.

\section{Discussion}

In a recent study [30], we proposed rehabilitation models of mechanisms, preventions and treatments of amyotrophic lateral sclerosis (caused by toxic invasions) that is related dementia disease [31]. In that study [30], we recommend to use warm running water with massages, without side effects, in clinical trials. $\mathrm{H} 2 \mathrm{O}$ plays a key role in the previous models. In this article, we suggest to use health oriented lifelong learning (HOLL) to prevent, delay and/or treat aging or aged dementia (caused by less mentally stimulating activities), without side effects, in clinical trials. O2 plays a key role in HOLL.

Generally, we think: $\mathrm{O} 2$ and $\mathrm{H} 2 \mathrm{O}$ are respectively the first and second important nutrients/maters to our lives; they are respectively critical for our bio-metabolism for every minute(s) and hour(s); most of nutrition supplies come from our normal drinking and eating.

However, we should emphasize that HOLL is moderate or mild challengeable and intensive, interesting, not very hard; over learning (mental exercises) will hurt our brains (clinical side effects), just like over physical exercises hurt our bodies.

Though we focus on cases of LTM in this study, we think the principles of our models are suitable to that of intermediate and short term memories (ITM and STM) .

We feel to learn or review textbooks together with our own children is not only encouraging them to study, but also is very effective to refresh or update our old memories; we also think to learn knowledge of health is very useful and significant for us to live healthily.

HOLL is not only favourable to aging or aged people and creative thinkers but also benefitting for job hunters. Based on our model in (Figure 4), we can infer why or how science or engineering trained people can do some literature jobs; while literature trained people almost cannot do any scientific or engineering work, usually and relatively. Therefore, we strongly suggest (young) people to study sciences, engineering and foreign languages as much as they can, to be successful job hunters as well as completely or versatilely educated learners.

\section{Conclusion}

Our health oriented lifelong learning (HOLL) models elucidate that moderate education, learning new knowledge, and performing moderate challengeable health oriented mental activities have not side effects and can be the first choices to prevent, delay and/or treat (onsets of) the aging or aged dementias caused by less mentally stimulating activities in clinical trials.

The principle of our models is, how we can acquire the memories, we can keep or retain them: use health oriented lifelong learning (HOLL) to build up and maintain multiple correlated memories in different knowledge fields.

Our models of specific and distributed signals and responses respectively explain mechanisms of music (or other arts) and electric therapies to treat dementias in the clinical researches.

\section{Authorship and contributor ship}

Kang Cheng made 1) substantial contributions to conception and design, analysis and interpretation of data; 2) drafting the article and revising it critically for important intellectual content; 3 ) final approval of the version to be published; and 4) agreement to act as guarantor of the work (ensuring that questions related to any part of the work are appropriately investigated and resolved).

Vivien Cheng made 1) substantial contributions to conception and design, analysis and interpretation of data; 2) revising it critically for important intellectual content; 3) final approval of the version to be published; and 4) agreement to act as guarantor of the work (ensuring that questions related to any part of the work are appropriately investigated and resolved).

Changhua Zou made the same contributions as Vivien Cheng did.

\section{References}

1. Ali GC, Guerchet M, Wu YT, Prince M, Prina M (2015) Chapter 2, The globa prevalence of dementia, In: World Alzheimer Report. The global impact of dementia. London: Alzheimer's disease international (ADI).

2. Alzheimer's Association (2018) Alzheimer's disease facts and figures. Alzheimer Dement 14: 367-429.

3. Fitzpatrick AL, Kuller LH, Ives DG, Lopez OL, Jagust W, et al. (2004) Incidence and prevalence of dementia in the cardiovascular health study. J Am Geriatr Soc 52: $195-$ 204. [Crossref]

4. Kukull WA, Higdon R, Bowen JD, McCormick WC, Teri L, et al. (2002) Dementia and Alzheimer disease incidence: a prospective cohort study. Arch Neurol 59: 1737-1746. [Crossref]

5. Evans DA, Bennett DA, Wilson RS, Bienias JL, Morris MC, et al. (2003) Incidence of Alzheimer disease in a biracial urban community: Relation to apolipoprotein $\mathrm{E}$ allele status. Arch Neurol 60: 185-9. [Crossref]

6. Stern Y (2012) Cognitive reserve in ageing and Alzheimer's disease. Lancet Neurol 11: 1006-1012. [Crossref]

7. Sando SB, Melquist S, Cannon A, Hutton M, Sletvold O, et al. (2008) Risk-reducing effect of education in Alzheimer's disease. Int J Geriatr Psychiatry 23: 1156-1162. [Crossref]

8. Grzywacz JG, Segel-Karpas D, Lachman ME (2016) Workplace Exposures and Cognitive Function During Adulthood: Evidence From National Survey of Midlife Development and the O*NET. J Occup Environ Med 58: 535-541. [Crossref]

9. Pool LR, Weuve J, Wilson RS, Bültmann U, Evans DA, et al. (2016) Occupationa cognitive requirements and late-life cognitive aging. Neurology 86: 1386-1392. [Crossref]

10. Then FS, Luck T, Luppa M, Arélin K, Schroeter ML, et al. (2014) Association between mental demands at work and cognitive functioning in the general population - results of the health study of the Leipzig research centre for civilization diseases (LIFE). J Occup Med Toxicol 9: 23. [Crossref]

11. Fisher GG, Stachowski A, Infurna FJ, Faul JD, Grosch J, et al. 2014. Mental work demands, retirement, and longitudinal trajectories of cognitive functioning. $J$ Occup Health Psychol 19: 231-42.

12. Department of Education and Science (2000) Learning for life: Paper on Adult Education. Dublin: Stationery Office.

13. Fang R, Ye S, Huangfu J, David P. Calimag DP (2017). Music therapy is a potentia intervention for cognition of Alzheimer's Disease: a mini-review. Translational Neurodegeneration 6: 2.

14. Junga JY (2017) The effect of single trial transcutaneous electrical nerve stimulation on balance and gait function in elderly people with dementia: a pilot study. Phys Ther Rehabil Sci 6: 59-64.

15. Cameron MH, Lonergan E, Lee H (2003) Transcutaneous Electrical Nerve Stimulation (TENS) for dementia. Cochrane Database of Systematic Reviews 3: CD004032.

16. Alladi S, Bak TH, Duggirala V, Surampudi B, Shailaja M, et al. (2013) Bilingualism delays age at onset of dementia, independent of education and immigration status. Neurology 81: 1938-1944. [Crossref] 
Cheng K (2018) Modeling health oriented lifelong learning (HOLL) to prevent, delay and/or treat aging or aged dementias caused by less mentally stimulating activities

17. Gold BT (2013) Lifelong bilingualism maintains neural efficiency for cognitive control in aging. Journal of Neuroscience 33: 387-396.

18. Cheng K (2012) Biomedical Infophysics. North Charleston, SC: CreateSpace Independent Publishing Platform.

19. Cheng K, Zou C (2007) Biomedicine and informatics model of Alzheimer's disease. Am J Biochem. Biotechnol 3: 145-149.

20. Cheng K, Zou C, (2010) Bio info physics models of neuronal signal processes based on theories of electromagnetic fields. American Journal of Neuroscience 1: 13-20.

21. Cheng K, Zou C, (2010) Information models of acupuncture analgesia and meridian channels. Information 1: 153-168.

22. Cheng K, Zou C, (2011) Biomedical info physics models of meridian channel system Webmed Central Biophysics 2: WMC002555.

23. Michalski RS, Carbonell JG, Mitchell TM (Eds.) 1983. Machine Learning, An Artificial Intelligence Approach. Tioga, CA: Springer-Verlag Berlin Heidelberg, Jointly published with Tioga Publishing Company.
24. Morris RG (1999) D.O. Hebb: The organization of behavior, Wiley: New York; 1949. Brain Res Bull 50: 437. [Crossref]

25. Hagan M (1996) Neural Network Design. New Dehli: PWS.

26. Hopfield JJ (1982) Neural networks and physical systems with emergent collective computational abilities. PNAS 79: 2554-2558.

27. Kapit W, Macey RI, Meisami E (1987) The Physiology Coloring Book. New York: Harper \& Row.

28. Simons JS, Spiers HJ (2003) Prefrontal and medial temporal lobe interactions in longterm memory. Nat Rev Neurosci 4: 637-648. [Crossref]

29. Ochoa G, Corey M (1995) The Timeline Book of Science. New York: Stonesong Press

30. Kang Cheng (2018) Rehabilitation models of mechanisms, preventions and treatments of amyotrophic lateral sclerosis caused by toxic invasions, Webmed Central Rehabilitation 9: WMC005502.

31. Zarei S, Carr K, Reiley L, Diaz K, Guerra O, et al. (2015) A comprehensive review of amyotrophic lateral sclerosis. Surg Neurol Int 6: 171. [Crossref]

Copyright: (C2018 Cheng K. This is an open-access article distributed under the terms of the Creative Commons Attribution License, which permits unrestricted use, distribution, and reproduction in any medium, provided the original author and source are credited. 